\title{
La reforma del Código Civil portugués respecto al estatuto del animal
}

\section{Alexandra Reis Moreira}

Abogada portuguesa especializada en Derecho Animal.

Miembro del Grupo de trabajo especializado en Derecho animal

del Centro de Estudios del Colegio de Abogados portugués.

Fundadora de Jus Animalium y experta consultora internacional en Derecho Animal

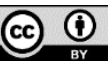

Recepción: Mayo 2018

Aceptación: Junio 2018

\section{Resumen}

La reciente modificación del Código Civil portugués, operada por la Ley nº/2017, fue el resultado de una larga, persistente y creciente demanda social de un tratamiento jurídico adecuado para los animales, a través de varias peticiones populares a los órganos de soberanía, con énfasis especial en la última década. En este artículo propongo analizar el proceso social y parlamentario que permitió el histórico cambio jurídico del estatuto de los animales en el Código Civil portugués con el objetivo de que sea replicado en otras latitudes, adaptando la dogmática jurídica a la realidad natural y social. Un cuestión, esta, que es de justicia.

Palabras clave: reforma del Código Civil portugués, estatuto de los animales, proceso parlamentario

Abstract. The reform of the Portuguese Civil Code regarding the status of the animals

The recent modification of the Portuguese Civil Code, operated by Law n ${ }^{\circ} 8 / 2017$, was the result of a long, persistent and growing social demand for an adequate legal treatment for animals, through several popular petitions to organs of sovereignty, with special emphasis on the last decade. In this article I propose to analyze the social and parliamentary process that allowed the historical legal change to the status of animals in the Portuguese civil code, in order to be replicated in other latitudes, adapting legal dogmatics to natural and social reality. Question, this, which is of justice.

Keywords: Animal law, mistreatment, crime, dogs, cats, serial killer, animal conscience. 


\section{ANTECEDENTES}

La reciente modificación del Código Civil portugués, operada por la Ley nº 8/2017 de 3 de marzo $^{1}$, fue el resultado de una larga, persistente y creciente demanda social de un tratamiento legal adecuado para los animales, a través de varias peticiones populares a órganos de soberanía, con especial énfasis en la última década ${ }^{2}$.

En efecto, ya en 2008 por iniciativa del Gobierno, a través del Ministerio de Justicia, se había nombrado un grupo de trabajo destinado a la elaboración de una Propuesta de Ley de modificación del Código Civil con el mismo objetivo de autonomizar jurídicamente a los animales de las "cosas".

En el período comprendido entre 2008 y el 2009 se consultó sobre esta cuestión a diversas entidades, en particular a asociaciones de protección animal, pero también a biólogos, veterinarios y juristas.

De acuerdo con lo hecho público por la asociación ANIMAL, el proyecto de ley en cuestión fue dirigido, en concreto, a añadir al Código Civil una disposición que decía lo siguiente: "los animales son seres sensibles, aunque pueden ser objeto de relaciones jurídicas, no son cosas y su protección se activa por ley especial ${ }^{3 \text { ”. }}$. Es decir, se preconizaba una fórmula similar a la vigente en otros países como Alemania, Austria y Suiza, que simplemente proclamaba que los animales no son $\operatorname{cosas}^{4}$. Sin embargo, este proyecto no llegó a salir adelante ${ }^{5}$.

A la vista de lo expuesto, el 03-02-2011 se dio entrada en la Asamblea de la República (el parlamento portugués) una petición de iniciativa popular, la Petición $n^{\circ} 138$ / XI / 2, suscrita por 8305 ciudadanos, con vistas a la "aprobación de la propuesta para la modificación del estatuto jurídico de los animales en el Código Civil presentada por el Ministerio de Justicia ${ }^{6 "}$. Lo que los peticionarios querían era la modificación del Código Civil con el fin de diferenciar a los animales de las cosas y reconocer su propia naturaleza de seres vivos y sensibles, tal y como los propios peticionarios aclararon el 14-09-2011, en la audiencia en el Parlamento ${ }^{7}$. Una vez que dicha petición reunía más de 4000 firmas, la misma

${ }^{1}$ Disponible en https://dre.pt/application/file/a/106551507
${ }^{2}$ Como ha señalado acertadamente el diputado a la Asamblea Nacional por el Partido Popular (CDS-
PP), João Rebelo, en un debate parlamentario celebrado el 21.10.2011, "las asociaciones de protección
de animales, los movimientos de protección de los animales, son los que presentan más peticiones en
este Parlamento y con el mayor número de firmas (...). No estamos hablando de algo que no es
importante, pero de algo que dice mucho a decenas de miles de personas que cada año recurren a la Asamblea de la República para presentar propuestas sobre estas materias"-cfr.

http://debates.parlamento.pt/catalogo/r3/dar/01/12/01/034/2011-10-21?sft=true\#p42

${ }^{3}$ V. http://blogdaanimal.blogspot.pt/2009/05/ministerio-da-justica-avanca-com.html

${ }^{4}$ Aunque les sean subsidiariamente aplicables las normas que regulan las cosas, en todo lo que no sea contrario a la naturaleza misma de los animales.

${ }^{5}$ Ciertamente ha contribuido para el fracaso de la iniciativa el cese de funciones del gobierno de entonces (XVII Gobierno Constitucional) que estuvo en ejercicio del 12 de marzo de 2005 al 26 de octubre de 2009. Presidido por el Primer Ministro José Sócrates, disponía de apoyo parlamentario mayoritario del Partido Socialista (PS). El Gobierno siguiente, liderado por el mismo Primer Ministro (ya muy debilitado políticamente, con el PS ganó las elecciones con mayoría relativa), y contando con otro Ministro de Justicia, conoció un corto y atribulado mandato, que culminó con su dimisión; en funciones desde el 26 de octubre de 2009 hasta el 21 de junio de 2011, fecha en que tomó posesión, después de elecciones, el XIX Gobierno Constitucional liderado por el Primer Ministro Pedro Passos Coelho, con apoyo parlamentario mayoritario, resultante de la alianza entre el Partido Social Demócrata (PSD), ganador de las elecciones con mayoría relativa y el Partido Popular (CDS-PP).

${ }^{6} \mathrm{Cfr}$.

http://app.parlamento.pt/webutils/docs/doc.pdf?path=6148523063446f764c324679626d56304c334e70 6447567a4c31684a5447566e4c31526c65485276526d6c75595778515a5852705932396c6379396b4e4 441345a6d51774f4330794e5749794c5452684d4759744f4759315a69316a4d7a63795a545133596a4e6 a5a6a67756347526d\&fich=d408fd08-25b2-4a0f-8f5f-c372e47b3cf8.pdf\&Inline=true

${ }^{7}$ Sobre todo porque la "propuesta del Ministerio de Justicia" no había pasado de un mero proyecto que Derecho Animal. Forum of Animal Law Studies, vol. 9/3 
fue tramitada en el plenario parlamentario, tal como se impone en la letra a) del apartado 1 del artículo 24 de la Ley n ${ }^{\circ} 43 / 90$, de 10 de agosto, con la redacción que le fue dada por la Ley no 6/93, de 1 de marzo, ley que regula el ejercicio del Derecho de Petición expresamente reconocido por la Constitución de la República Portuguesa en su artículo 52. En consecuencia, se llevó a cabo el 21-10-2011 un fructífero debate parlamentario, con intervenciones de todos los grupos parlamentarios, en lo esencial concordantes con la revisión del estatuto de los animales, algunos de ellos, con intención de presentar Proyectos de Ley sobre este tema ${ }^{8}$. Escasos tres meses después, el 10-01-2012, entró en la Asamblea de la República una nueva petición de iniciativa popular, la Petición n ${ }^{\circ} 80$ / XII / 1, suscrita por 12393 ciudadanos, requiriendo el "cumplimiento del artículo 13 del Tratado de Lisboa, que Portugal firmó y ratificó, y el cambio consecuente e inmediato de los Códigos Civil y Penal, en la parte relativa a los animales, seres sintientes, no "cosas muebles ${ }^{9 "}$.

El 15-02-2012, el Partido Socialista presentó el primer Proyecto de Ley destinado a modificar el Código Civil para establecer un estatuto jurídico de los animales, y así se enuncia el Proyecto de Ley ${ }^{10} n^{\circ} 173$ / XII / 1.

Se trata de un texto con una redacción bastante aproximada a la que en 2016, fue acogida por el Proyecto de Ley presentado por el mismo grupo parlamentario y que dio origen a la Ley $n^{\circ} 8 / 2017$.

En síntesis y en lo esencial, el Proyecto de Ley n ${ }^{\circ} 173$ / XII / 1 preconizaba la adición de la norma 202 A, bajo el epígrafe "Animales", en la sección del Código Civil titulada "De las cosas", que pasaría a titularse, "De las cosas y de los animales". Es decir, aunque diferenciados de las cosas, los animales eran mantenidos en el mismo subtítulo que regulaba las cosas, no teniendo autonomía sistemática.

Además, se optó por una definición de los animales, que únicamente establecía que los animales pueden ser objeto de relaciones jurídicas, remitiendo su protección a la legislación especial y previendo la aplicación subsidiaria de las normas relativas a las cosas ${ }^{11}$.

Ese Proyecto de Ley también proponía normas innovadoras ${ }^{12}$, en particular, la regulación del destino de los animales de compañía en los casos de divorcio y la exclusión de los animales de compañía del régimen matrimonial de comunidad universal de bienes. Se

nunca fue presentado en la Asamblea de la Republica (parlamento portugués). Cfr. http://app.parlamento.pt/webutils/docs/doc.pdf?path=6148523063446f764c324679626d56304c334e70 6447567a4c31684a5355786c5a793944543030764d554e425130524d5279394562324e3162575675644 7397a5547563061574e68627938345a6a4531595451334d6930314d6a677a4c54526c4f445974595752 694e53307a4f5463314d7a41344e47517a4e5751756347526d\&fich=8f15a472-5283-4e86-adb539753084d35d.pdf\&Inline=true

${ }^{8}$ Véase el debate en este registro : http://debates.parlamento.pt/catalogo/r3/dar/01/12/01/034/2011-1021/42?pgs=41-45\&org=PLC\&plcdf=true

${ }^{9}$ Disponible en:

http://app.parlamento.pt/webutils/docs/doc.pdf?path=6148523063446f764c324679626d56304c334e70 6447567a4c31684a5355786c5a7939555a58683062305a70626d46735547563061574e765a584d76595 75a6d4e7a4d7a4f4755744d7a677a4f5330304d7a63354c5749794d32497459324d78595451344e7a517 9597a55794c6e426b5a673d3d\&fich=aff7338e-3839-4379-b23b-cc1a48742c52.pdf\&Inline=true

${ }^{10}$ Disponible en:

http://app.parlamento.pt/webutils/docs/doc.pdf?path=6148523063446f764c3246795a5868774d546f33 4e7a67774c336470626d6c7561574e7059585270646d467a4c31684a535339305a58683062334d76634 770734d54637a4c56684a5353356b62324d3d\&fich=pil173-XII.doc\&Inline=true

Este proyecto de ley tiene como su primer suscriptor el diputado Pedro Delgado Alves, ampliamente conocido por su compromiso personal y político con la causa animal, entre otras.

${ }^{11}$ El artículo 202-A, titulado "Animales", tenía el siguiente contenido:

1. Los animales pueden ser objeto de relaciones jurídicas, y la protección jurídica derivada de su naturaleza opera por vía de ley especial.

2 - A los animales se les aplican las disposiciones relativas a las cosas sólo cuando la ley especial no sea aplicable y sólo en la medida en que no sean incompatibles con el espíritu de ella.

${ }^{12}$ Lo que llegó a ser contemplado cinco años más tarde, a través de la Ley nº 8/2017, de 3 de marzo. 
pidieron dictámenes sobre el Proyecto de Ley en cuestión al Consejo Superior del Ministerio Público y al Consejo Superior de la Magistratura ${ }^{13}$, los cuales no presentaron ninguna objeción a las soluciones propuestas ${ }^{14}$.

Dada la coincidencia de tema, se programó para el 29-03-2012 la discusión conjunta de la Petición ${ }^{\circ} 80$ / XII / 1 y del Proyecto de Ley n 173 / XII / 1. Los registros de ese debate parlamentario dan cuenta de las profundas diferencias de fondo sobre el asunto, anticipándose el probable rechazo de la Ley en la votación designada para el día siguiente ${ }^{15}$.

Ante el posible naufragio de la iniciativa legislativa, el grupo parlamentario suscrito propuso con éxito que el Proyecto de Ley en crisis fuera remitido, sin votación, por un período de 90 días, a la comisión parlamentaria especializada competente en razón de la materia, la Comisión de Asuntos Constitucionales, Derechos, Libertades y Garantías, con vistas a la profundización de los trabajos de reflexión de la temática en cuestión y a la búsqueda de soluciones de compromiso.

Por carta de 17-07-2015, la citada Comisión dio a conocer al plenario que no había sido posible la adopción de un texto consensual de sustitución y solicitando, en los términos reglamentarios, la programación de la votación del Proyecto de Ley, en su versión originaria.

En consecuencia, temiendo los efectos nefastos del previsible rechazo del Proyecto de Ley, los diputados suscriptores del mismo solicitaron su no inclusión para votación. Después de un recorrido parlamentario accidentado, el Proyecto de Ley $\mathrm{n}^{\circ} 173$ / XII / 1 , acabaría por caducar el 22-10-2015, por efecto del término de la legislatura en curso. Precisamente el mismo año que en Francia, después de un intenso debate nacional que duró un año, la Asamblea Nacional reformaba el Código Civil, reconociendo a los animales como seres vivos dotados de sensibilidad ${ }^{16}$.

${ }^{13}$ Equivalentes, respectivamente, al Ministerio Fiscal y al Consejo General del Poder Judicial en España.
${ }^{14}$ El Consejo del Ministerio Público (Fiscalía) concluyó que el contenido del proyecto de ley era
coherente con los principios filosóficos, éticos, políticos y culturales enunciados en el mismo; El Consejo
Superior de la Magistratura (Poder Judicial), sugirió, además, que el reconocimiento de un estatuto
jurídico de los animales debía ser seguido por la reflexión sobre otros temas relacionados con el ejercicio
del derecho de propiedad sobre los animales, en particular, adoptando un régimen claro de la tenencia
de animales de compañía en propiedad horizontal. Los dictámenes de que se trata pueden consultarse a través de estos links:

http://app.parlamento.pt/webutils/docs/doc.pdf?path=6148523063446f764c324679626d56304c334e70 6447567a4c31684a5355786c5a793944543030764d554e425130524d5279394562324e3162575675644 7397a5357357059326c6864476c3259554e7662576c7a633246764c3245315a47457959324e6a4c544e6 95a5749744e474d324d693169596a566b4c574d344d6a4d774e6d56695954566b596935775a47593d\&f ich=a5da2ccc-3beb-4c62-bb5d-c82306eba5db.pdf\&Inline=true

http://app.parlamento.pt/webutils/docs/doc.pdf?path=6148523063446f764c324679626d56304c334e70 6447567a4c31684a5355786c5a793944543030764d554e425130524d5279394562324e3162575675644 7397a5357357059326c6864476c3259554e7662576c7a633246764c3255354e6d49344f4755304c57566 b596d55744e44526c5a4330344d7a5a6a4c546b314d5749354f4467344d7a526d4f4335775a47593d\&fi ch=e96b88e4-edbe-44ed-836c-951b988834f8.pdf\&Inline=true

${ }^{15}$ Se recuerda que la mayoría parlamentaria era, en ese momento, PSD / CDS-PP. La bancada del grupo parlamentario de PSD sostuvo que el proyecto de ley era insuficiente, meramente simbólico y que, en un momento en que la ley penal no protegía los animales (excepto aquellos que fueran propiedad, a través del delito de daños) la prioridad debía ser proteger a los animales, en particular los animales de compañía. A su vez, el CDS-PP se ha esforzado por argumentar que las modificaciones propuestas sólo tendrían sentido para proteger a los animales de compañía, y agregó que "no se puede aplicar igualmente a otros animales", además de ser un cambio con mero valor simbólico, solo el Derecho Público podría garantizar la efectiva protección. Por su parte, el Partido Comunista (PCP) expresó que esta es una "herramienta reducida y de dudoso significado práctico" y que la prioridad debe ser la implementación de la legislación existente y garantizar su aplicación. Sólo los grupos parlamentarios de la minoría parlamentaria, el Bloque de Izquierda (BE) y Los Verdes han mostrado concordancia con las iniciativas en discusión - cfr. registros de las intervenciones parlamentarias: http://debates.parlamento.pt/catalogo/r3/dar/01/12/01/090/2012-03-30/29?pgs=29-36\&org=PLC

${ }^{16}$ Aunque sujeta a la misma disciplina de las cosas y inserta en el capítulo relativo a los "bienes". La 


\title{
2. CUESTIONES TÉCNICO-JURÍDICAS PLANTEADAS DURANTE LOS TRABAJOS PARLAMENTARIOS
}

El 04.15.2016, ya en una nueva legislatura y con una base de apoyo parlamentario mayoritario ${ }^{17}$, el Partido Socialista presenta otro Proyecto de Ley, con $\mathrm{n}^{\mathrm{o}} 164$ / XIII, con fecha 14/04/2016 y con el mismo objeto del pionero nº173 / XII /1.

En esencia, se mantienen las normas sobre los animales en la sección de las cosas, cuyo título se extendió a los objetos y los animales y se adopta el concepto de animales importado del Código Civil francés ${ }^{18}$ :

\author{
Libro I - Parte General \\ (...) \\ Título II - De las relaciones jurídicas \\ Subtítulo I - De las personas \\ (...) \\ Subtítulo II - De las cosas y de los animales \\ (...) \\ Artículo 202-A \\ (Animales)
}

1. Los animales son seres vivos dotados de sensibilidad, operando la protección jurídica derivada de su propia naturaleza por vía de legislación especial.

2. A los animales se les aplica subsidiariamente las disposiciones relativas a las cosas, en ausencia de una ley especial.

Además del citado Proyecto de Ley, también se presentaron otros tres Proyectos de ley sobre la misma materia:

a) El Proyecto de Ley n 171 / XIII / de modificación del Código Civil reconociendo a los animales como seres sensibles, presentado por el Partido PersonasAnimales-Naturaleza (PAN) ${ }^{19}$ de 15.4.2016.

b) El Proyecto de Ley n ${ }^{\circ} 224$ / XIII, que modifica el estatuto jurídico de los animales

modificación del Código Civil francés, que tuvo mucha proyección en la opinión pública también en Portugal, fue editado por la Ley ${ }^{\circ}{ }^{\circ}$ 2015-177 del 16/02/2015, que introdujo el nuevo artículo 515-14 del Libro II, titulado "De los bienes y las diferentes modificaciones de la propiedad https://www.legifrance.gouv.fr/affichCode.do;jsessionid=399A6B66C5160DC251F760D2DDE1925C. tpdila14v 2?idSectionTA=LEGISCTA000006090204\&cidTexte=LEGITEXT000006070721\&dateTe xte $=20170520$

${ }^{17}$ En base a tres acuerdos firmados bilateralmente entre el Partido Socialista (PS) y los otros tres partidos de izquierda, Bloque de Izquierda (BE), Partido Comunista Portugués (PCP) y Partido Ecologista "Los Verdes" (PEV), que también viabilizaron la constitución del XXI Gobierno Constitucional, que tomó posesión a 26-11-2015.

${ }_{18}$ El Proyecto de Ley $\mathrm{n}^{0} 164$ / XIII se puede encontrar en: http://app.parlamento.pt/webutils/docs/doc.pdf?path=6148523063446f764c3246795a5868774d546f33 4e7a67774c336470626d6c7561574e7059585270646d467a4c31684a53556b76644756346447397a4c3 34271624445324e43315953556c4a4c6d527659773d3d\&fich=pjl164-XIII.doc\&Inline=true

${ }^{19}$ Partido político cuyos estatutos anuncian la "protección, defensa y armonización justa de los derechos de los animales humanos, de los animales no humanos y de la naturaleza, presentes y futuros" - cf. https://www.pan.com.pt/comunicacao/noticias/item/393-estatutos.html - y que por primera vez desde su constitución en 2009 logró elegir a un diputado en las elecciones legislativas de octubre de 2015. El parlamento portugués está compuesto por 230 diputados. Esta elección es sintomática de la creciente movilización de los ciudadanos por la protección de los animales, votando en un partido político que se afirma defensor de esa causa y la naturaleza, presente y futuro". Se puede encontrar en: https://www.parlamento.pt/ActividadeParlamentar/Paginas/DetalheIniciativa.aspx?BID=40235 
en el Código Civil, presentado por el Partido Social Demócrata (PSD) el 06/05/2016 ${ }^{20}$.

c) El Proyecto de Ley n 227 / XIII / que reforma el Código Civil asignando un estatuto jurídico a los animales, presentado por el Bloque de Izquierda (BE) el $06 / 05 / 2016^{21}$.

Recibidos los dos Proyectos de Ley presentados el 04/15/2016, se remitieron a la Comisión Parlamentaria de la especialidad, que solicitó dictámenes al Colegio de Abogados $^{22}$, al Consejo Superior de los Jueces y al Consejo Superior del Ministerio Público.

Tanto el Consejo Superior de los Jueces como el Colegio de Abogados expresaron su preferencia por las soluciones más completas y técnicamente mejores del Proyecto de Ley $n^{\circ} 164$ / XIII 26. Sin embargo, esta última entidad, opinó que la disposición sistemática de las normas sobre los animales debía ser autónoma de las cosas. Así las cosas, el 12-05-2016 tuvo lugar la discusión parlamentaria conjunta de los cuatro citados Proyectos de Ley.

El diputado socialista Pedro Delgado Alves, recordó que el tema en debate ya no era nuevo, tenía antecedentes parlamentarios y que ya otros países europeos como Alemania, Austria, Suiza y más recientemente Francia, ya habían aplicado el mismo cambio en sus Códigos Civiles, "sin que ello hubiese acarreado ningún drama y de modo que, en relación con los animales, se sigue aplicando la legislación del sector agropecuario, en las materias de la cría, de las especies protegidas, de animales en espectáculos, de investigación científica y de tratamiento sanitario "; Y, por lo tanto, que lo que estaba en juego era sólo diferenciar a los animales de las cosas. Es decir, acertadamente optó por un discurso apaciguador de los temores manifestados hace años por las industrias citadas y sus representantes parlamentarios.

A continuación se enunciaron las posiciones de los grupos parlamentarios que hubieron expresado menor apertura a la reforma del estatuto jurídico de los animales ${ }^{23}$ :

El Partido Socialdemócrata (PSD), afirmó que la "percepción de que los animales no eran cosas ya estaba de alguna manera implícita en los cambios realizados en la ley penal" en $2014^{24}$, por lo que también se había presentado un Proyecto Ley por coherencia con el

\footnotetext{
${ }^{20}$ Se puede encontrar en:

http://app.parlamento.pt/webutils/docs/doc.pdf?path=6148523063446f764c3246795a5868774d546f33 4e7a67774c336470626d6c7561574e7059585270646d467a4c31684a53556b76644756346447397a4c3 34271624449794e43315953556c4a4c6d527659773d3d\&fich=pjl224-XIII.doc\&Inline=true

${ }^{21}$ El Proyecto de Ley no 171 / XIII /1 (PAN) recibió más críticas, en su mayoría relacionadas con la discapacidad técnica, inconsistencia jurídica y la insuficiencia de las normas propuestas, en concreto, dejando abiertas las cuestiones clave tales como el derecho de propiedad de los animales; del lado positivo, el dictamen emitido por el Colegio de Abogados destacó su mejor opción traducida en la automatización sistemática de las normas generales sobre los animales en relación con las cosas, como también propuesto por el Bloque de Izquierda (BE) a través de su proyecto de ley No. 227 / XIII / 1.Se puede encontrar en:

http://app.parlamento.pt/webutils/docs/doc.pdf?path=6148523063446f764c3246795a5868774d546f33 4e7a67774c336470626d6c7561574e7059585270646d467a4c31684a53556b76644756346447397a4c3 34271624449794e79315953556c4a4c6d527659773d3d\&fich=pj1227-XIII.doc\&Inline=true

${ }^{22}$ El Colegio de Abogados portugués tiene competencia sobre todo el territorio nacional.

${ }^{23}$ Las intervenciones parlamentarias se pueden encontrar en:

http://debates.parlamento.pt/catalogo/r3/dar/01/13/01/067/2016-05-12/16?pgs=8$15 \&$ org=PLC\&plcdf=true

${ }^{24}$ Se recuerda que, en 2014, se había aprobado la Ley nº 69/2014 de 29 de agosto, después de un largo debate parlamentario, que modificó el Código Penal portugués, introduciendo dos nuevos tipos de delitos: el delito de maltrato y el delito de abandono, ambos restrictos a los animales de compañía. Esta ley ha inaugurado en Portugal, la tutela penal, aunque restringido a los animales de compañía. Hasta entonces, las conductas de abuso y abandono de animales de compañía constituían mera infracción administrativa.
} 
tratamiento jurídico de los animales. Con interés en aplacar los temores de algunos sectores económicos, adelantó que su partido no aceptaría que "esos proyectos o cualquiera otros alteren o afecten a las actividades económicas, a las actividades agrícolas, a las actividades cinegéticas u otras que se derivan de tradiciones profundamente arraigadas en el pueblo portugués ${ }^{25 " .}$.

A su vez, el Partido Comunista (PCP) hizo hincapié en la "necesidad de una mayor reflexión" sobre las cuestiones pendientes, expresando su preocupación por algunos conceptos sugeridos como la "conciencia de los animales ${ }^{26 "}$ y sus consecuencias.

El Partido Popular (CDS-PP) por su parte, subrayó la necesidad de tener "mucha cautela" en estas materias, ya que el Código Civil "es el instituto jurídico fundamental de las relaciones jurídicas privadas". A continuación, planteó que "la cuestión central es ésta: ante el régimen jurídico de las cosas, ¿deben o no los animales tener un estatuto propio, autónomo? ¿O deben ser considerados como un tertium genus, dentro del marco legal?", agregando que no tienen nada para oponerse a esta idea. En cuanto a la iniciativa legislativa de BE, hizo hincapié en la insuficiencia de la definición de los animales como sintientes ${ }^{27}$, cuestionando qué animales son sintientes ${ }^{28}$. Y finalizó su discurso afirmando que nunca aceptarían Proyectos de Ley sirvieran para" prohibir la caza, prohibir la ganadería o incluso prohibir las corridas de toros", señalando que no es eso que estaba en cuestión ${ }^{29 ”}$.

A continuación, el diputado que fue el primer suscriptor del Proyecto de Ley n ${ }^{0} 164$ / XIII, Pedro Delgado Alves, hizo la síntesis del debate, concluyendo que existe una zona de consenso parlamentario en torno a la creación de una categoría específica adecuada a los animales y tranquilizó precisando que la modificación sería sólo en el ámbito del Código Civil, manteniendo el resto del marco legal.

Los Proyectos de Ley fueron entonces remitidos a la comisión parlamentaria de la especialidad, donde fue creado un grupo de trabajo específico y temporal para instruir en ese asunto $^{30}$ y promover la reflexión y la búsqueda de consensos, titulado "Grupo de Trabajo sobre las iniciativas legislativas sobre el Bienestar Animal ${ }^{31}$ ".

En ese marco, se escucharon diversas entidades, en particular, representantes de asociaciones de protección animal y de los médicos veterinarios, profesores universitarios y representantes de otros sectores económicos relacionados con los animales.

\footnotetext{
${ }^{25}$ Obviamente, en referencia a las corridas de toros.

${ }^{26}$ Referencia evidente al concepto de animales sintientes propuesto en el Proyecto de Ley $n^{\circ} 227$ / XIII / 1(BE).

${ }^{27}$ El Proyecto de Ley no 227 / XIII /1 (BE) sugiere el siguiente concepto a los animales a través de un nuevo artículo 201-B, cuyo contenido nos parece demasiado complejo, denso e inadecuado para los objetivos del Código Civil:

1 - Los animales sintientes no humanos tienen valor en sí mismos y son dignos de protección jurídica, que se realiza, en particular, en el conjunto de obligaciones de los poseedores legales estipulado en el artículo siguiente.

2 - Son animales sintientes los que tienen la capacidad de percibir conscientemente lo que les rodea y de recibir y reaccionar a los estímulos de forma consciente, incluyendo emociones positivas y negativas.

${ }^{28}$ De hecho, la elección de esta definición científica - que ni siquiera cumple con el consenso de la neurociencia con respecto a algunos animales - causaría necesariamente dificultades al intérprete común de la norma (y pudendo perjudicar los intereses de los propios animales).

29 Discurso que ha resultado ser una contribución importante para apaciguar a los sectores que cuestionaban el cambio que se pretendía, cuyos intereses son siempre defendido por el partido político en cuestión.

${ }^{30}$ Pero también otras iniciativas legislativas en curso relacionadas con la ampliación y el fortalecimiento de la protección penal de los animales, que fueron rechazadas, meses más tarde, en la misma fecha en que se ha aprobado el cambio del Código Civil (22-12-2016).

${ }^{31}$ Este grupo de trabajo se compuso de siete miembros representando a todas las fuerzas políticas presentes en el Parlamento y desarrolló su actividad del 08.06.2016 al 01.05.2017, cuyo registro se puede encontrar en:
}

https://www.parlamento.pt/sites/COM/XIIILEG/1CACDLG/GTILDA/Paginas/Composicao.aspx 
Por ser de mayor interés, dado que traducen obstáculos a la iniciativa en cuestión, veamos en particular las posiciones de estos últimos. En resumen, los representantes de la agricultura / ganadería, caza y pesca, cunicultura, creación del caballo Lusitano y de la tauromaquia, han expresado diversas preocupaciones sobre este asunto, en particular ${ }^{32}$ :

- La supuesta indefinición del término "sensibilidad".

- La oposición al reconocimiento de los animales como "titulares de intereses jurídicamente protegidos ${ }^{33 "}$.

- La supuesta vaguedad de la expresión "sin justa causa" norma adoptada para vedar al dueño de un animal "sin causa legítima, infligir dolor, sufrimiento o cualquier otra enfermedad, un tratamiento que resulta en un sufrimiento innecesario, el abandono o la muerte" del animal ${ }^{34}$.

- Incertidumbre resultante de la norma ${ }^{35}$ que se refiere a protección de los animales señalada como "ley especial" cuyo contenido es desconocido.

- La referencia a las "formas inadecuadas de entrenamiento ${ }^{36 "}$ debe suprimirse, por crear inseguridad jurídica.

Finalmente, todos estos aspectos cuestionados fueron debidamente aclarados por los diputados presentes. En concreto, que los aparentes conceptos indefinidos e indeterminados serían concretados por los tribunales, caso por caso, como es habitual en la práctica jurídica; que la imputación de los malos tratos tendría que resultar de una actividad humana intencionada, y que la legislación especial es la legislación ya en vigor sobre la materia, no estando prevista la adopción de la nueva disciplina jurídica.

Como resultado de los trabajos desarrollados por la comisión respectiva, el Grupo parlamentario del PS presentó propuestas de modificación a los textos de los Proyectos de Ley n 164 / XIII (PS), n 171 / XIII (PAN), nº 224 / XIII (PSD) y n 227 / XIII (BE), en forma de un solo texto de sustitución de todos estos proyectos, que recibió la aprobación unánime de los diputados restantes ${ }^{37}$.

En lo esencial, el referido texto de sustitución aprobado acogió las soluciones normativas materiales preconizadas en el Proyecto de Ley $\mathrm{n}^{\circ} 164$ / XIII (PS), pero dando autonomía a las normas que definen los animales y el estableciendo su estatuto jurídico en un subtítulo específico, sistemáticamente situado entre el subtítulo - De las personas y el subtítulo - De las $\operatorname{cosas}^{38}$. Se ha optado por no incluir ningún Preámbulo ni Exposición de

\footnotetext{
${ }^{32}$ Audiencias a las que se puede acceder en: http://www.canal.parlamento.pt/?cid=1185\&title=audiencia-da-confederacao-dos-agricultores-deportugal-cap http://www.canal.parlamento.pt/?cid=1191\&title=audicao-do-clube-portugues-de-caniculturaassociacao-nacional-de-prop

${ }^{33}$ Propuesto en el proyecto de ley No. 171 / XIII /1 (PAN).

${ }^{34}$ Propuesto por el apartado 2 del artículo 1305o-A del proyecto de ley No. 164 / XIII (PS). Señaló como ejemplos la posibilidad de animales utilizados en la actividad pecuaria que se dejen a pastar al calor intenso o que se queden atascados en resultado de fuertes lluvias, lo cual, según los consultados, puede ocurrir fácilmente.

${ }^{35}$ Artículo 202-A, apartados 1 y 2 del proyecto de ley No. 164 / XIII (PS).

${ }^{36}$ Prevista en el proyecto de ley No. 224 / XIII (PSD) como conducta prohibida frente a los animales por los respetivos propietarios.

${ }^{37} \mathrm{El}$ informe del trabajo realizado se puede encontrar en:

http://app.parlamento.pt/webutils/docs/doc.pdf?path=6148523063446f764c324679626d56304c334e70 6447567a4c31684a53556c4d5a5763765130394e4c7a464451554e45544563765247396a6457316c626e 527663306c7561574e7059585270646d46446232317063334e68627938355a4451784d6a49314f53316 b4e7a5a6a4c5452685a4759744f444a684d7930304f446b784e3255774e6d45324e6a6b756347526d\&fi ch=9d412259-d76c-4adf-82a3-48917e06a669.pdf\&Inline=true

${ }^{38}$ Solución que había sido propuesta por los proyectos de ley presentados por el BE y el PAN.
} 
Motivos $^{39}$

El 22-12-2016, dicho documento fue aprobado por unanimidad en la Asamblea de la República, dando origen a la Ley $n^{\circ}$ 8/2017 de 3 de marzo, de modificación del Código Civil, del Código de Proceso Civil y del Código Penal ${ }^{40}$.

\section{LAS MODIFICACIONES FINALES INTRODUCIDAS POR LA REFORMA E INICIO DE VIGENCIA}

La Ley n ${ }^{\circ}$ 8/2017 fue publicada el 3 de marzo de 2017 y entró en vigor el 1 de mayo de 2017. En el artículo 1 establece su objeto que no es otro que el establecimiento de un estatus jurídico de los animales, reconociendo su naturaleza de seres vivos dotados de sensibilidad. A continuación, se enuncian las modificaciones introducidas al Código Civil y al Código de Procedimiento Civil:

\section{- Adopción de normas específicas para los animales:}

a) Se añadió un nuevo subtítulo -I A- De los animales, dispuesto de forma sistemática entre el subtítulo I- De las Personas y el subtítulo II -De las $\operatorname{cosas}^{41}$, en el que se incluyeron las siguientes normas:

"Subtítulo I-A - De los animales

Artículo 201-B Animales

Los animales son seres vivos dotados de sensibilidad y objeto de protección jurídica en virtud de su naturaleza.

Artículo 201-C Protección jurídica de los animales

La protección jurídica de los animales opera a través de las disposiciones del presente Código y de legislación especial.

Artículo 201-D Régimen subsidiario

En ausencia de ley especial, se aplicarán subsidiariamente a los animales las disposiciones relativas a las cosas, siempre que no sean incompatibles con su naturaleza.

(...)”

b) De lo expuesto se concluye que se ha acogido la solución francesa en la definición que se da a los animales ${ }^{42}$ y que, en ausencia de normas específicas, son aplicables las normas que regulan las cosas en todo lo que no sea incompatible con la naturaleza de los animales. Cabe señalar que, en la práctica, la mayoría de las normas que regulan el derecho de propiedad y los negocios

\footnotetext{
${ }^{39}$ Posiblemente evitándose consideraciones de contenido fracturante.

${ }^{40}$ A pesar de que los proyectos de ley destinados a reforzar la protección penal han sido rechazados, era necesario adaptar los tipos penales que protegen la propiedad al cambio civil, a través de la adición de los animales a estas normas penales (que sólo se referían a las "cosas").

${ }^{41}$ Por lo tanto, en términos sistemáticos la proximidad resultó aún mayor con las personas que con las cosas, aunque, como se verá, el régimen material dominante sigue siendo lo de las cosas.

${ }^{42}$ Como expendemos en el dictamen presentado por el Colegio de Abogados, habría sido más correcto, en nuestro entendimiento, adoptar la fórmula los animales son seres vivos dotados de sensibilidad y de sistema nervioso, ya que es este último elemento que distingue a los animales de las plantas - cf. (P 12 y

ss.):http://app.parlamento.pt/webutils/docs/doc.pdf?path=6148523063446f764c324679626d56304c334 e706447567a4c31684a53556c4d5a5763765130394e4c7a464451554e45544563765247396a6457316c6 26e527663306c7561574e7059585270646d46446232317063334e68627938334d3251315a4468695a69 30314e7a63334c5451795a6a6b744f44637a5a5330784e6a45784d5755794d5749794e7a6b756347526d \&fich=73d5d8bf-5777-42f9-873e-16111e21b279.pdf\&Inline=true
} 
jurídicos también se aplican a los animales.

\section{- En relación a la responsabilidad civil por actos ilícitos}

Se añade el artículo 493-A, que pasa a establecer expresamente:

a) El derecho a la indemnización a cargo del responsable de dañar a un animal por los gastos de tratamiento en que su propietario o poseedor /cuidador (sea persona física o colectiva) haya incurrido, aunque sean de valor superior al que se haya atribuido al animal.

b) El derecho a una indemnización por daños personales al propietario en caso de lesión al animal de compañía que resulte en la privación de órgano o miembro importante o la afectación grave y permanente de su capacidad de locomoción ${ }^{43}$.

\section{- En materia de regulación del derecho de propiedad}

a) Se introduce un nuevo número ${ }^{44}$ a la norma que define el "objeto del derecho de propiedad" (artículo 1302), disponiendo que los animales pueden ser objeto del derecho de propiedad, en los términos regulados en ese código y en la legislación especial.

b) Se añade el artículo 1305-A, relativo a la propiedad de animales", con el siguiente contenido ${ }^{45}$ :

“Artículo 1305-A Propiedad de animales

1. El propietario de un animal debe asegurar su bienestar y respetar las características de cada especie, y observar en el ejercicio de sus derechos las disposiciones especiales relativas a la cría, reproducción, tenencia y protección de los animales y a la salvaguardia de especies en riesgo siempre que sea necesario.

2. A los efectos del apartado anterior, la obligación de garantizar el bienestar incluye, en particular:

a) la garantía de acceso a agua y alimentación de acuerdo con las necesidades de la especie en cuestión.

b) la garantía de acceso a cuidados médico-veterinarios siempre que esté justificado, incluidas las medidas profilácticas, de identificación y de vacunación previstas por la ley.

3. El derecho de propiedad de un animal no incluye la posibilidad de, sin motivo legítimo, infligir dolor, sufrimiento o cualquier otro maltrato del que resulte sufrimiento injustificado, abandono o muerte.”

c) El nuevo artículo 1305-A enuncia limitaciones generales al derecho de propiedad de los animales y se distingue de la norma que le precede y que dispone sobre la

\footnotetext{
${ }^{43}$ Hay que señalar que este derecho ya había sido establecido por la jurisprudencia, como resultado de las normas generales que regulan el derecho a la reparación por actos ilícitos - cf. Sentencia del Tribunal de Apelación de Oporto, de 19.02.2015, disponible en:

http://www.dgsi.pt/jtrp.nsf/56a6e7121657f91e80257cda00381fdf/3c0d5d98d088fab880257dfc00556b d1? OpenDocument

${ }^{44} \mathrm{El}$ apartado 1 se refiere a las cosas muebles e inmuebles.

${ }^{45}$ Fue el resultado de un compromiso entre los proyectos de ley del PS (artículo 1305.o-A), PSD (artículo 1305.o, apartados 2 y 3) y BE (artículo 201-C, apartado 3).
} 
propiedad de las cosas (artículo 1305), reconociendo en esto caso los derechos plenos de uso, frutos y la disposición de éstos.

d) Se aclara la distinción entre los animales y las cosas en la norma ${ }^{46}$ que regula el instituto de la ocupación (artículo 1318):

“Artículo 1318 Susceptibilidad de ocupación

Pueden ser adquiridos por ocupación los animales y las cosas móviles que nunca tuvieron dueño, o fueron abandonados, perdidos o escondidos por sus propietarios, guardando las restricciones de los artículos siguientes.”

e) Se elimina el anacrónico, inútil y desde hace mucho tiempo criticado artículo 1321, debido a los juicios de valor sobre el carácter de los animales en cuestión, que mantenía su redacción originaria de 1966, con la epígrafe "animales feroces huidos” y que disponía que "los animales feroces y maléficos que se evaden de la clausura en que su dueño los tenga pueden ser destruidos u ocupados libremente por cualquier persona que los encuentre”. Se ha de señalar que ninguno de los cuatro Proyectos de Ley preveía la revocación de ese artículo, sino sólo la alteración de su redacción. Sin embargo, el dictamen del Colegio de Abogados sobre esta materia, indicado anteriormente, subrayaba en su página 14, que esa norma era inútil y lo que preveía podía resolverse recurriendo a otras normas que ya figuraban en el código civil.

f) En las normas referidas a los animales y cosas muebles perdidas (artículo 1323) se diferencia a los animales de las $\operatorname{cosas}^{47}$, así como se mejora la redacción de la misma y se consagra el derecho de retención o incautación del animal encontrado en el caso de que haya fundado temor de que el mismo sea víctima de malos tratos por parte del propietario.

\section{- En el ámbito del derecho de familia se efectuaron cambios en relación con los animales de compañía}

a) Se excluyeron de la sociedad ganancial a los animales de compañía que cada uno de los cónyuges tenía antes de casarse - artículo 1733, párrafo 1, col. h).

b) - se ha imposto la obligación de ponerse de acuerdo sobre el destino de los animales de compañía en caso de divorcio por consentimiento mutuo - artículo 1775, apartado 1, letra h);

c) - en el caso de divorcio (sin consentimiento mutuo) el tribunal confiará a los animales de compañía a uno o ambos cónyuges, teniendo en cuenta en particular los intereses de cada uno de ellos y de los niños de la pareja y también el bienestar del animal (así establece el nuevo artículo 1793-A).

\section{- En relación con el Código de Procedimiento Civil}

Se cambió el artículo 736 respeto la tramitación de la ejecución, declarando que los

\footnotetext{
${ }^{46}$ La redacción original era la siguiente: Pueden ser adquiridos por ocupación los animales y otras cosas móviles que nunca tuvieron dueño, o fueron abandonados, perdidos o escondidos por sus propietarios, guardando las restricciones de los artículos siguientes.

${ }^{47} \mathrm{La}$ anterior redacción de la norma se refiere a los animales u otro móvil perdido (los relieves en el texto son nuestros).
} 
animales de compañía son absolutamente inembargables, lo que se traduce en una pertinente y realmente útil innovación jurídica con importantes efectos sociales ${ }^{48}$.

\section{CONCLUSIONES}

1) El reciente proceso parlamentario portugués, que comenzó en 2016, tras el reconocimiento de un estatuto jurídico civil adecuado para los animales a través del cambio del Código Civil, no fue sinuoso ni difícil. En ello han contribuido diversos factores conjugados entre sí:

a) La experiencia portuguesa en esa materia gracias a diversas y muy participadas peticiones populares presentadas desde hacía años y a los impulsos legislativos llevados a cabo desde 2008, por iniciativa del Partido Socialista portugués.

b) La composición actual del parlamento portugués, con mayoría de apoyo al gobierno socialista.

c) El consenso en este ámbito de los operadores jurídicos, manifestado a través del Colegio de Abogados, el Consejo Superior de los Jueces y el Ministerio Fiscal.

d) La actualidad del tema en la escena internacional, en particular por la reforma del Código Civil francés y, en el plano nacional, por el hecho cierto de que en 2014 se introdujo por primera vez la tutela penal de los animales, aunque limitada a los animales de compañía. También es de resaltar que desde entonces se vienen multiplicando las iniciativas académicas en forma de conferencias, cursos, jornadas sobre Derecho Animal.

2) Los principales obstáculos al cambio de la Ley fueron presentados por representantes de intereses económicos como las industrias pecuarias, las de la creación y utilización de animales en actividades deportivas y lúdicas, las de los caballos lusitanos y de la tauromaquia. Han defendido su postura argumentado la falta de necesidad de reformar el Código Civil, alegando la suficiencia de las normas ya existentes de protección de los animales. Cabe resaltar, que no obstante lo anterior, gracias a la habilidad política y el pragmatismo de algunos diputados como el socialista Pedro Delgado Alves, fue posible aplacar los temores mostrados, sin dejar de avanzar con las soluciones consideradas justas.

3) Los cambios introducidos en el Códigos Civil y, simultáneamente en el Código de Procedimiento Civil, representan un paso histórico en el sistema legal portugués en lo que concierne a la consideración de los animales, principalmente porque representa el abandono de conceptos y nociones no sólo anacrónicos y desajustados a la realidad e incoherentes con el resto del Ordenamiento Jurídico vigente en Portugal.

A pesar del avance, en nuestra opinión, la reforma acaecida en Portugal, no deja de ser un paso necesario pero tímido, ya que a pesar de dotar a los animales de un estatuto jurídico civil propio, todavía sigue siendo subsidiariamente aplicable a los animales el régimen de las $\operatorname{cosas}^{49}$.

\footnotetext{
${ }^{48}$ Cambio que se aplaude y que no se encontraba en cualquiera de los cuatro Proyectos de Ley.

${ }^{49}$ De la misma manera nos hemos pronunciado en el dictamen emitido por el Colegio de Abogados portugués, antes citado.
} 